\title{
Research on Transient Electromagnetic Detection Method for Substation Site Selection
}

\author{
Yang Bo ${ }^{1,}{ }^{*}$, Wang Qian $^{1}$, Xu Zhengy ${ }^{2}$, and Bao Minghui ${ }^{1}$ \\ ${ }^{1}$ Equipment Condition Evaluation Center, State Grid Chongqing Electric Power Research Institute, 401123 Chongqing, China \\ ${ }^{2}$ The School of Electrical Engineering, Chongqing University, 400044 Chongqing, China
}

\begin{abstract}
A transient electromagnetic detection method for site selection of substation grounding is proposed in this paper. And taking the fault detection experiment of transmission lines in a certain area of China as an example, the transient electromagnetic technology is used to obtain high-quality images reflecting the development trend and distribution of geological faults. The results show that the transient electromagnetic method is a convenient and effective way for substation site selection.
\end{abstract}

\section{Introduction}

The stability of power supply has attracted much attention, and landslides around the substation pose a threat to the safety of power transmission, so it is urgent to carry out high-resolution detection and evaluation of geological structures near the surface. The common method to find out the landslide overburden and sliding surface is to drill or dig holes by hand at a certain interval in the landslide area. It requires a large amount of engineering, cost, and time. Using geophysical methods to determine the morphological characteristics of the landslide has the advantages of less time and relatively low cost. The traditional geophysical methods for landslide surveys include the seismic method, resistivity method, and electromagnetic method ${ }^{[1]}$. Among them, the seismic method has relatively serious damage to the environment, and the detection depth with resistivity method is shallow although it can detect the internal structure and sliding surface of the landslide ${ }^{[2-3]}$. Moreover, the geological $\operatorname{radar}^{[4]}$ is conducive to detecting shallow sliding surfaces and cracks, but its detection depth is limited owing to the electromagnetic waves attenuated by saturated water or clay areas ${ }^{[5]}$. Inspired by the fact that the resistivity value can be used as the detection index of landslide deformation and startup $^{[6-7]}$, the imaging technology of transient electromagnetic apparent resistivity has a good prospect in the field of geological disaster prediction in substations.

Transient Electromagnetic Method (TEM) is a geophysical exploration method for detecting underground geoelectric information, which is based on the principle of electromagnetic induction. To be specific, through an ungrounded loop (transmitter coil), an electromagnetic pulse is sent to the underground. Under the excitation of the primary field, eddy currents are induced inside the underground conductive geological body. During the gap between electromagnetic pulses, the secondary magnetic field generated by the induced eddy current does not disappear immediately with the disappearance of the primary field, where exists a transient process. The response signal of the secondary field is observed by the receiving coil, and the variation of that with time is studied, and then the electrical parameters and spatial distribution of the underground conductive geological body are determined ${ }^{[8]}$. The electromagnetic and humanistic interference of the substation is strong, and the construction area is narrow. Moreover, the terrain has a great influence on the substation on the landslide body. While, the small wireframe transient electromagnetic method has the characteristics of simple construction, convenient use, and strong shallow detection ability, which is suitable for the detection and construction of substation landslide. Therefore, the high-resolution detection of near-surface geological structures has become a frontier problem of $\operatorname{TEM}^{[9-10]}$

TEM is sensitive to low-resistivity geological bodies and is especially suitable for detecting aquifers of landslides. There are obvious differences in electromagnetic parameters between loose accumulations, bedrock, and air in landslide cracks, with different electrical conductivity. The landslide fracture development zone has strong permeability, low medium water content, irregular distribution of fracture voids in space, high bedrock resistivity, and the distribution law of landslide body can be detected by transient electromagnetic apparent resistivity imaging. Therefore, the use of transient electromagnetic to detect landslide cracks has sufficient geophysical premises.

In this paper, a transient electromagnetic detection method is proposed for substation location selection, and the imaging theory of the transient electromagnetic apparent resistivity of that is analysed. Moreover, an on- 
site testing experiment is carried out in Ganzi Tibetan Autonomous Prefecture, Sichuan Province.

\section{Principle of Transient Electromagnetic Method}

\subsection{Basic principles of transient electromagnetic method}

The transient electromagnetic detection device is mainly composed of a transmitter, transmitting coil, receiver, and receiving coil. The working process is divided into three parts: transmitting, electromagnetic induction, and receiving. And its schematic diagram is shown in Fig. 1.

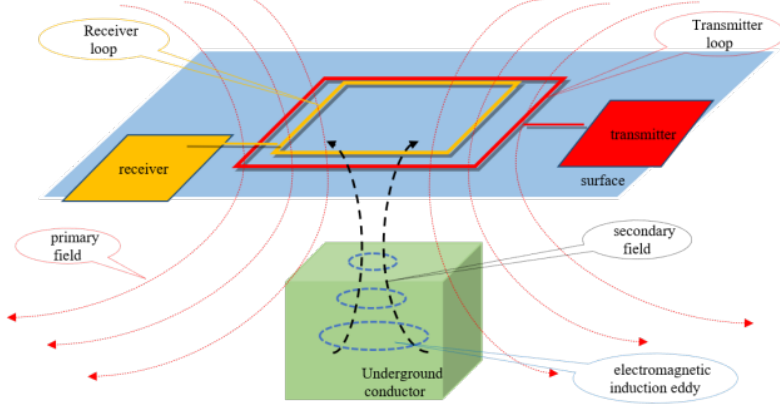

Fig. 1 Principle of the transient electromagnetic method

According to Faraday's law of electromagnetic induction, when the stable current in the transmitting coil is suddenly turned off, an induced current will be generated in the underground well-conducting geological body.

The diffusion process of induced eddy current in the geological body can be divided into three stages: early, middle, and late ${ }^{[11]}$. In the early stage, the highfrequency components are dominant because of the highfrequency effect of skin depth preventing the electromagnetic field from propagating to the deep underground. Thus, the electromagnetic field mainly reflects shallow geological information. With the heat loss of induced current in the geological body, the surface current begins to diffuse into the geological body, and the diffusion velocity of that is generally inversely proportional to the conductivity, which is called the middle stage. In the late stage, the effect of lowfrequency components in the geoelectric medium is relatively obvious, where the electromagnetic field reflects the deeper geological information, and the current distribution in the geological body tends to be stable. Meanwhile, the attenuation speed of the secondary magnetic field generated by the induced current is greatly reduced. The magnitude of the transient response at this time mainly depends on the longitudinal conductance of the ground section ${ }^{[12]}$.

\subsection{Transient electromagnetic resistivity imaging method}

apparent

The American geophysicist Nabigian believes that the transient electromagnetic response produced by the induced eddy currents above the earth's surface is the total effect of various eddy layers underground.

This effect can be approximated by the equivalent of current loops propagating downwards. These current loops are like "smoke rings" blown out by the transmitting coil, the shape of which is the same as that of the transmitting coil. As time increases, the current loop expands outward and downward continuously. Therefore, the process of vortex diffusion is visually called the "smoke ring effect"[13]. Fig. 2 shows the process of underground diffusion and propagation of "smoke ring"[14-15]. The transient electromagnetic response of the induced eddy current above the earth's surface can be approximated by a current loop, and its radius increase and depth deepen as time increases. Therefore, when calculating the transient electromagnetic response of the ground, the current loop at a certain time can be equivalently used to replace the eddy current, and the "smoke ring" can be regarded as a series of secondary transmitting coils, so that it is easy to calculate the change rule of the response value with time for a measuring point. In layered media, there is still a "smoke ring" effect, but the propagation of the "smoke ring" is gradually limited to conductive strata ${ }^{[16]}$.

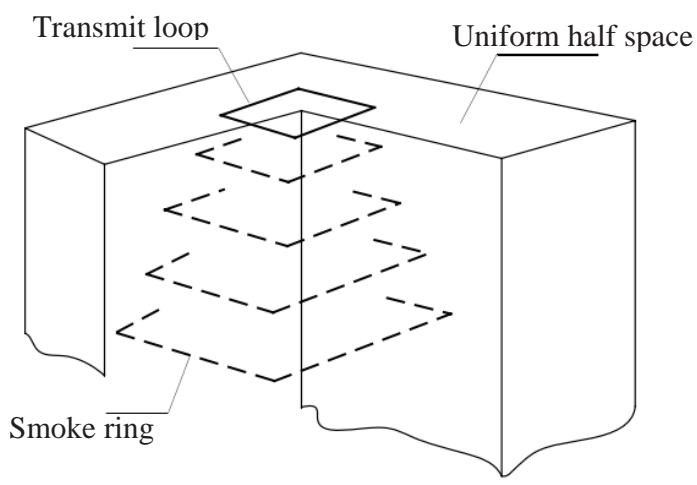

Fig. 2 Schematic diagram of the "smoke ring" theory

According to the theory of "smoke ring", the negative step response excited by the transmitting coil on the ground surface of uniform half-space, can be approximated by a "smoke ring". The calculation formula of vertical depth $\left(d_{r}\right)$ and diffusion radius $\left(R_{r}\right)$ of "smoke ring" at a certain time is as follows ${ }^{[12]}$.

$$
\begin{gathered}
d_{r}=\frac{4}{\sqrt{\pi}} \sqrt{\frac{\tau \rho_{0}}{\mu_{0}}} \\
R_{r}=a+2.091 \sqrt{\frac{\tau \rho_{0}}{\mu_{0}}}
\end{gathered}
$$

The vertical propagation speed of the "smoke ring" is:

$$
v=\frac{2}{\sqrt{\pi}} \sqrt{\frac{\rho_{0}}{\mu_{0} t}}
$$


The apparent depth corresponding to the apparent resistivity is:

$$
H_{r}=0.441 \frac{\left(d_{r_{i}}+d_{r_{j}}\right)}{2}
$$

In equations (1) to (4), $a$ is the radius of the transmitting coil, $\rho_{0}$ is the resistivity of the uniform halfspace, $t$ is the sampling time, and $\mu_{0}$ is the vacuum permeability; $d_{\mathrm{ri}}$ and $d_{\mathrm{rj}}$ are the vertical depths corresponding to $t_{\mathrm{i}}$ and $t_{\mathrm{j}}$ respectively, where $t_{\mathrm{i}}$ and $t_{\mathrm{j}}$ represent the sampling time of two adjacent detection channels $(j>i)$. It can be seen from equation (1) to equation (4) that the speed of the outward and downward diffusion of induced eddy current is related to the resistivity of the underground medium, that is, the better the conductivity of the medium is, the slower the diffusion speed will be. This means that the response signal can be observed on the ground after a long delay when the conductivity of the underground medium is good. From the perspective of the "smoke ring effect", the transient electromagnetic response signal in the early stage was generated by the induced eddy current near the ground surface, reflecting shallow geoelectric information; while the transient electromagnetic response signals in the late stage are mainly caused by the deep induced eddy current, which reflects deep geoelectric information. Therefore, it is possible to detect the geoelectric information that the earth changes vertically by studying the temporal variation of transient electromagnetic response signals.

Most transient electromagnetic instruments are used to measure the induced voltage of the receiving coil. To avoid the problem of multiple solutions when calculating the apparent resistivity in the attenuation section of the central loop ${ }^{[17-18]}$, it is necessary to convert the induced voltage into the magnetic field at first. The method of converting the induced voltage into a magnetic field is introduced in literature ${ }^{[19-21]}$. In this paper, the trapezoidal pre-estimation correction method with higher precision is used, where the initial value is estimated by the Euler method and corrected by the trapezoidal integral method.

In the central loop mode of the transient electromagnetic method, the vertical component $B_{\mathrm{z}}$ of the secondary field at the central of the receiving coil can be expressed as ${ }^{[22]}$ :

$$
B_{z}=\frac{\mu I}{2 a}\left[\frac{3}{\sqrt{\pi} u} e^{-u^{2}}+\left(1-\frac{3}{2 u^{2}}\right) \operatorname{erf}(u)\right]
$$

Where $a$ is the radius of the transmitting coil, $I$ is the steady-state value before the transmission current is turned off, $\mu$ is the permeability of the uniform halfspace, $\operatorname{erf}(u)=\frac{2}{\sqrt{\pi}} \int_{0}^{u} e^{-t^{2}} d t$ is the error function, $t$ is the time parameter calculated from the time when the transmitting current is switched off, $u=a / 2 \sqrt{\mu / \rho_{0} t}$ is the transient field parameter, $\rho_{0}$ is the resistivity of the uniform half-space. Then the apparent resistivity can be expressed as:

$$
\rho(t)=\frac{a^{2}}{4 t u^{2}}
$$

Equation (2.6) is workable during the entire transient process, so $\rho(t)$ defined here is called the overall apparent resistivity. And equation (2.5) can be expressed as follows with the parameter $u$ :

$$
B_{z}=\frac{\mu I}{2 a} Y(u)
$$

Where $Y(u)$ is the kernel function of $B_{\mathrm{z}}$, and

$$
Y(u)=\left[\frac{3}{\sqrt{\pi} u} e^{-u^{2}}+\left(1-\frac{3}{2 u^{2}}\right) \operatorname{erf}(u)\right]
$$

Therefore, the method for solving the overall apparent resistivity defined by equation (6) is as follows: the measured induced voltage is converted into magnetic induction intensity at first, and then $u$ value conforming to equation (7) is obtained by equation (8). Finally, $u$ is substituted into formula (6) to get the corresponding apparent resistivity curve $\rho(t)$.

Through the above analysis, the visual depth and visual resistivity information required for transient electromagnetic apparent resistivity imaging can be obtained.

\section{Karst detection experiment of the transient electromagnetic method in a certain area of china}

\subsection{Detection scheme of the transient electromagnetic method}

The artificial sources is used to excite the target to generate induced eddy currents for the transient electromagnetic method. The induced magnetic field during the pulse interval is generally obtained by measuring the induced electromotive force of the receiving coil.

A transient electromagnetic measurement system is constructed in this paper, and the transmitter of that adopts the high-power TZTEM-1 array-type shallow transient electromagnetic system, which is produced by Chongqing Cuilu Detection Technology Co., Ltd. To be specific, the transient electromagnetic emission system has the advantages of large emission current and highspeed linear shutdown. And the receiving and collecting system includes a variety of measurement, control and analysis modules, which is convenient for users to establish corresponding test and control models. 
Moreover, multiple acquisition channels are used to collect the induced voltage and transmit current of the receiving coil, where the maximum sampling rate is $4 \mathrm{MHz}$ and adopts 24-bit A/D.

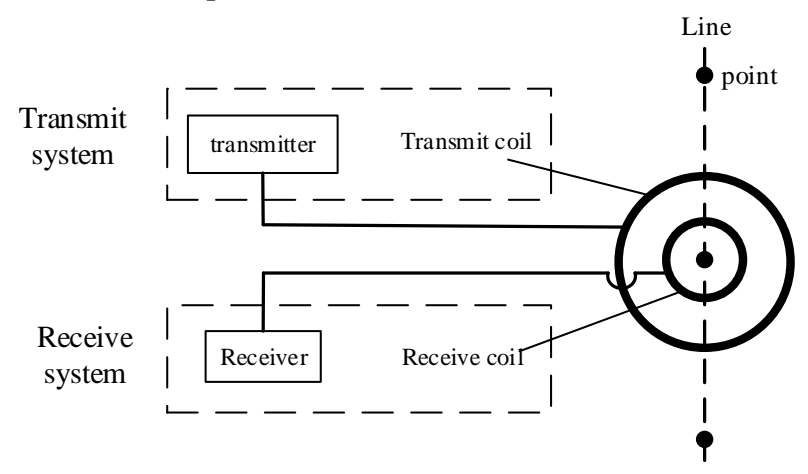

Fig.3 Transient electromagnetic data acquisition

The transient electromagnetic detection experiment system is shown in Fig. 3. A high-power transient electromagnetic transmitter provides a rapid turn-off oblique step current signal to the transmitting coil. The transient magnetic field generated by the turn-off current induces a secondary magnetic field through the eddy current effect of the underground low-resistance body and is collected by the receiving coil. Then use these data to obtain the visual resistivity imaging of the measurement point through the formula in section 2 of the article.

\subsection{Fault detection experiment of a transmission line in Luding city}

The survey line passes through the karst strata and is mainly composed of slightly weathered diorite. The rock structure is uneven, with locally mixed quartz diorite or diabase vein intrusion, accompanied by slight metamorphism. The rock mass is mainly fragmented and is affected by the structural fracture zone and the vein intrusion. The width of the intermittent distribution zone is 20-40 meters, and it is mostly in the mosaic fracture structure. Some sections have large karst bodies. Geological hazards such as karst collapse and karst foundation collapse caused by karst have brought great danger to the safety construction of substations and the guarantee of later operation quality.

According to the development trend and distribution of geological conditions, the survey area and survey line are delineated. There is one measuring line, and the schematic diagram of the experiment site is shown in Fig. 4.

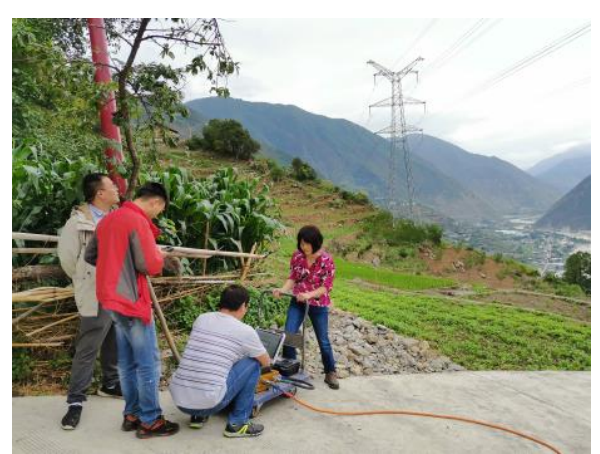

Fig. 4 Schematic diagram of the experiment site

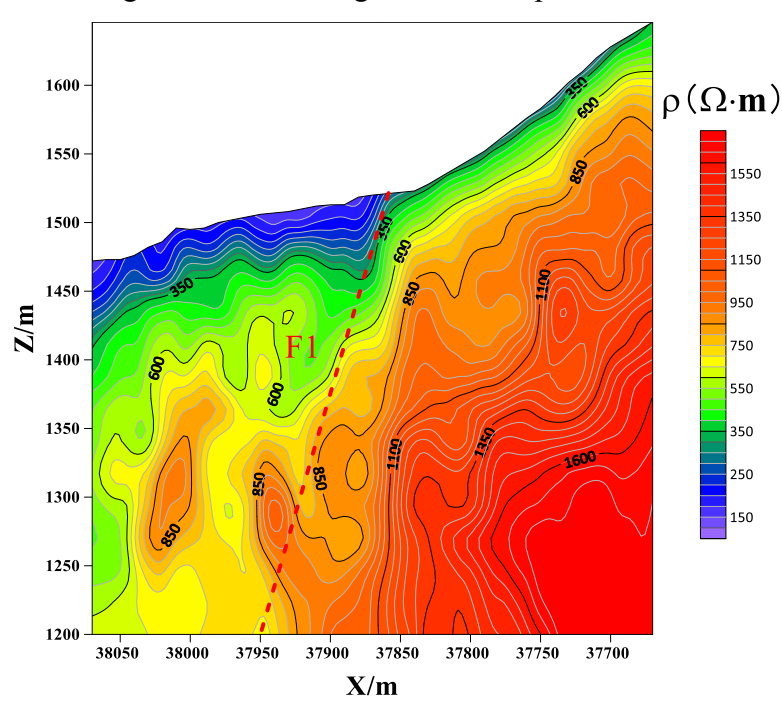

Fig. 5 Transient electromagnetic apparent resistivity imaging results

Fig.5 shows the apparent resistivity imaging of the cross section obtained through data processing. As can be seen from Fig.5, when the mileage is between 37850 and 38070 , the measuring location is located at the lower part of the hillside, and there is a covering layer of about 20 meters, while at the top of the hillside, the covering layer is thin. When the mileage is between 37850 and 37950 , it is speculated that there may be a right-inclined fault structure F1. Moreover, near the fault contact surface, there is a possibility of rock fragmentation, fissure development, water-rich layer, and karst development is higher.

\section{Conclusion}

Aiming at the characteristics of strong electromagnetic interference and humanistic interference in the substation and narrow construction area, a transient electromagnetic detection method is proposed for substation location selection in this paper. A fault detection experiment for transmission lines is carried out in a certain area in China, and high-quality images reflecting the location, direction, and depth of underground resistivity are obtained. Through comparison of on-site geological data, it is speculated that the low-resistance fault of the geological body has the possibility of forming a water-rich layer and karst. The characteristics of transient electromagnetic detection such as portability, fast, accurate, non-destructive, and intuitive image, especially the ability to work under harsh working conditions, will make it more widely used in the field of substation site geological survey.

\section{References}

1. $X u X Q$, Su L J, et al. Summary of research status of geophysical methods for detecting structural characteristics of landslide body [J]. Progress in Geophysics, 2015, 30(3): 1449-1458. 
2. Torgoev A, Lamair L, Torgoev I, et al. 2013. A review of recent case studies of landslides investigated in the Tien Shan using microseismic and other geophysical methods [A]. Earthquake Induced Landslides [M]. Berlin Heidel berg: Springer, 285-294

3. Qi Y L, Chen N S, Q in Q R, et al. Interpretation of ground penetrating radar images of sandstone and its slope deposits [J]. Journal of Chengdu University of Technology (Science technology edition), 2007, 34(1):97.

4. Li H, Lu G Y, He X Q, et al. Discussion on the development course and prospect of ground penetrating radar [J]. Progress in Geophysics, 2010, 25(4) : 14921502.

5. Bichler A, B obrowsky P, B est M, et al. Three-dimensional mapping of a landslide using a multi-geophysical approach: the Quesnel Forks landslide [J]. Landslides, 2004, 1(1):29-40.

6. Lapenna V, Lorenzo P, Perrone A, el al. 2D electrical resistivity imaging of some complex landslides in the L ucanian A penning chain, southern Italy [J]. Geophysics, 2005, 70(3):811-818.

7. M eric $0, G$ arambois $S$, J ongmans $D$, et al. A pplication of geophysical methods for the investigation of the large gravitational mass movement of Sechilienne,France [J]. Canadian G eotechnical J ournal, 2005, 42(4):1105-1115.

8. Pu H R. Principle of electromagnetic sounding [M]. Beijing: Geological Publishing House, 1990.

9. Ji Y J, Lin J, et al. Distortion analysis and numerical elimination of transient electromagnetic receiver for shallow detection [J]. Progress in Geophysics, 2007, 22(1): 263-265.

10. Niu Z L. Principle of time domain electromagnetic method [M]. Changsha: Central South University Press, 2007.

11. Jing $S$ J, Li Z D. Basic principles of transient electromagnetic method [J]. China Coalfield Geology, 1995, 7(2): 83-87.

12. Brian R. Spies. Depth of investigation in electromagnetic sounding methods. Geophysics, 1989, 54(7):872-887.

13. Jiang $B$ Y. Transient electromagnetic exploration [M]. Beijing: Geological Publishing House, 1998.

14. Sun $Y$ G, Tan D M. Three-dimensional numerical simulation of transient electromagnetic method under full space effect [J]. J ournal of Railway Engineering Society, 2010, 1(3):76-80.

15. Wang $Y Z, Y u J B, L i u$ J, et al. Transient electromagnetic method mine advance detection [J]. Chinese Journal of Engineering Geophysics, 2009, 6(1):28-32.

16. X ue G Q, Li X, Di Q Y. Research progress on forward and backward inversion of transient electromagnetic method [J]. Progress in Geophysics, 2008, 23(4): 11651172.

17. Xie L T. Transient electromagnetic rapid imaging [D]. Chongqing: Chongqing University, 2009.

18. Bai D H, Maxwell A M, Lu J, et al. Numerical calculation of the apparent resistivity in the whole process in the time domain transient electromagnetic method [J]. Chinese Journal of Geophysics, 2003, 46(7): 697-704.

19. Zhong S M, Zheng D Z. Transition process analysis [M ]. B eijing: Tsinghua University Press, 1986.
20. Chen $M$ S, Tian $X$ B. Study on transient electromagnetic sounding of galvanic source (5) The measured induced voltage is converted into vertical magnetic field [J]. Coal Geology \& Exploration, 1999, 27(5): 63-65.

21. Luo G P, Wei F Y. Study on Magnetic field of transient electromagnetic method and its apparent resistivity [J]. Coal Geology of China, 2000, $12(2): 60-62$.

22. Xie $L T, W u$ P. Numerical Calculation Method of Transient Electromagnetic Apparent Resistivity Bisection Pre-estimation [J]. Chinese Journal of Engineering Geophysics,2010, 7(5): 554-560. 